\title{
TUNING THE NARROW-BAND BEAM POSITION MONITOR SAMPLING CLOCK TO REMOVE THE ALIASING ERRORS IN APS STORAGE RING ORBIT MEASUREMENTS*
}

\author{
X. Sun ${ }^{\#}$ and O. Singh, ANL, Argonne, IL 60439, U.S.A.
}

\section{Abstract}

The Advanced Photon Source storage ring employs a real-time orbit correction system to reduce orbit motion up to $50 \mathrm{~Hz}$. This system uses up to 142 narrow-band $\mathrm{rf}$ beam position monitors (Nbbpms) in a correction algorithm by sampling at a frequency of $1.53 \mathrm{kHz}$. Several Nbbpms exhibit aliasing errors in orbit measurements, rendering these Nbbpms unusable in real-time orbit feedback. The aliasing errors are caused by beating effects of the internal sampling clocks with various other processing clocks residing within the BPM electronics. A programmable external clock has been employed to move the aliasing errors out of the active frequency band of the real-time feedback system (RTFB) and $\mathrm{rms}$ beam motion calculation. This paper discusses the process of tuning and provides test results.

\section{INTRODUCTION}

In order to stabilize the beam orbit in the band up to 50 $\mathrm{Hz}$, the Advanced Photon Source (APS) storage ring uses a real-time orbit feedback system [1]. This system uses up to 142 Nbbpms in the global correction algorithm with update rate at $1.53 \mathrm{kHz}$. Also, the rms beam motion value, provided to users in real time, is calculated in the frequency band of $0.017-30 \mathrm{~Hz}$ by using up to 80 of the above 142 Nbbpms. But some Nbbpms exhibit spikes in orbit measurements due to aliasing effects. Figure 1 shows plots of vertical cumulative rms motion data for several Nbbpms (A:P1s), taken during the operation of 24-bunch top-up mode with 102-mA beam, which exhibit steps. These aliasing effects make these Nbbpms unusable for both the real-time orbit feedback and rms beam motion calculation.

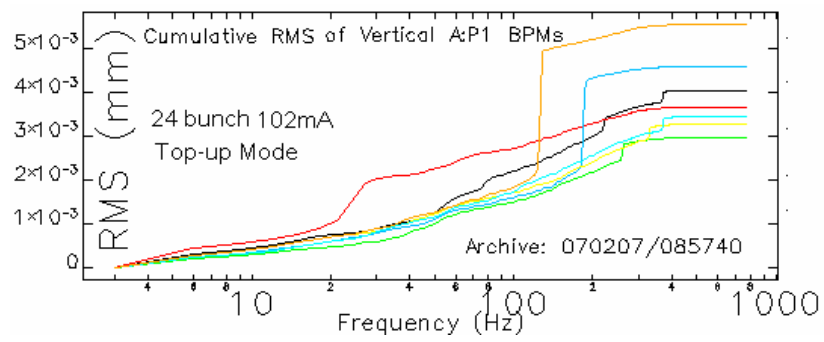

Figure 1: The cumulative rms data for several vertical Nbbpms show steps due to aliasing effects. Data is collected under 24-bunch top-up mode with 102-mA beam.

*Work supported by the U. S. Department of Energy, Office of Science, Office of Basic Energy Sciences, under Contract No. DE-AC0206CH11357.

\#xiang@aps.anl.gov
The aliasing effects are caused by the beating of the internal sampling clocks with various other processing clocks residing within the BPM electronics or from the adjacent BPM module. These are also related to the beam fill patterns. A commercial eight-channel VME-based sine/pulse generator card - PAS9782 (Precision Analog Systems) [2] - has been employed to provide a programmable external clock to override the internal clocks on the Nbbpm modules. At present, one clock is used to drive up to four Nbbpm modules. The external clock can be programmed to generate different frequency sine waves to move the aliasing errors out of the active frequency band of the real-time feedback system and the rms beam motion calculation.

\section{DRIFT MEASUREMENTS OF INTERNAL AND EXTERNAL CLOCKS}

We compared the stabilities between the internal clocks generated by each Nbbpm module and the external clock generated by the PAS9782 clock module.

\section{Internal Clocks Drifts for the Nbbpms}

The stabilities of the internal clocks on the Nbbpm modules were measured in storage ring sector 1 during an operation run. The temperature changes, as observed by the temperature sensors mounted on the outside of the module, were also measured. Figure 2 shows the data for all four Nbbpm modules, located in the same rack. The temperature drifts are up to 0.3 degree $\mathrm{C}$ for an 8-hour period. The internal clocks show drifts up to $2.5 \mathrm{~Hz}$, exhibiting correlation with temperature variations.

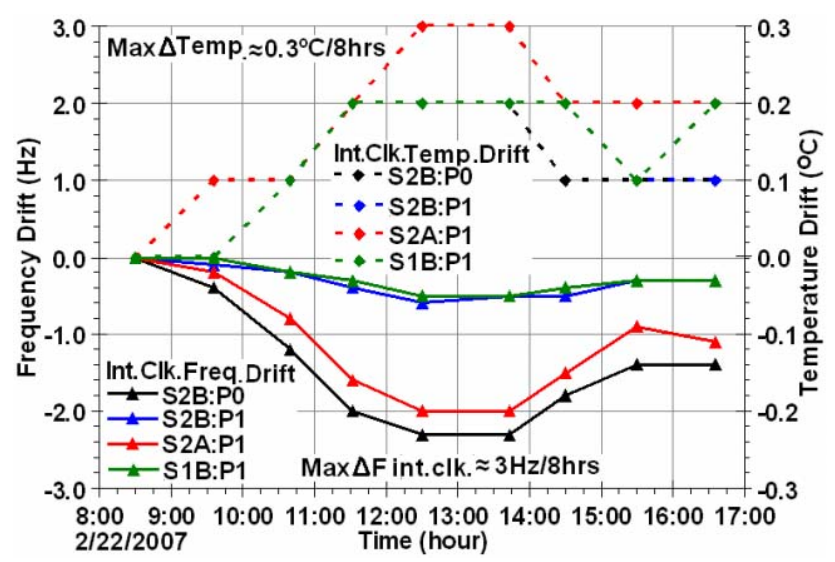

Figure 2: The internal clock drifts (solid lines) are correlated with temperatures (dashed lines). Nominal internal clocks are $\sim 32 \mathrm{kHz}$. 


\section{PAS9782 Module Clock Drift}

The stability of the external clock for the VME-based PAS9782 module was measured in the laboratory. Both the clock and temperature variations are shown in Figure 3.

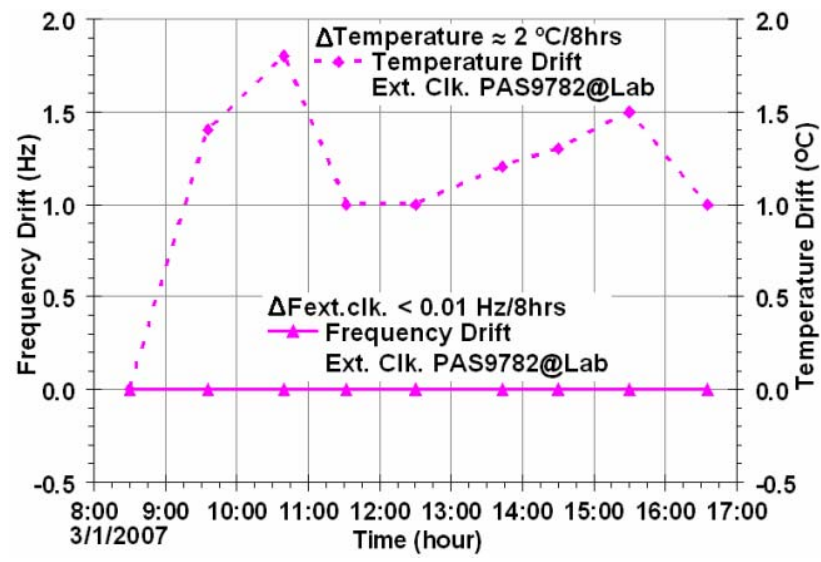

Figure 3: Plots of the external clock and the environment temperature drifts

Note that even with larger temperature variation (1.8 degree $\mathrm{C}$ ), the maximum drift of the external clock is only $\sim 0.01 \mathrm{~Hz}$ for an 8-hour period. The external clock is much more stable than the internal clocks on the Nbbpms modules.

\section{POSITION DATA EFFECTS WITH EXTERNAL CLOCK VARIATION}

The PAS9782 module was programmed by EPICS and applied as an external clock to four Nbbpm modules in one chassis in a laboratory environment. The external clock effects on the DC and AC position measurements are discussed here.

\section{Effects}

We measured the DC data of the four Nbbpm modules as the external clock varied from $36 \mathrm{kHz}$ to $39.2 \mathrm{kHz}$ in $400 \mathrm{~Hz}$ steps. Data are shown in Figure 4.

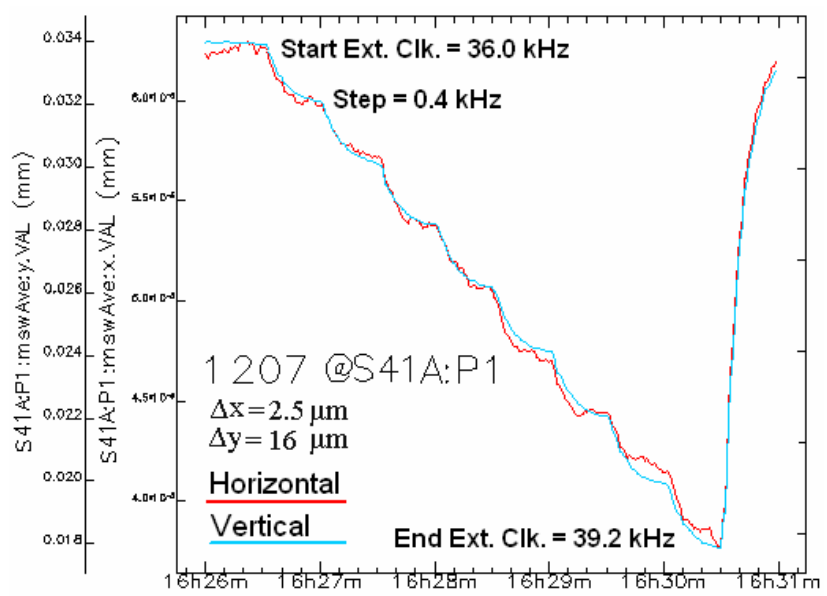

Figure 4: Horizontal and vertical position data changes are shown as the external clock varies.

06 Instrumentation, Controls, Feedback \& Operational Aspects
The external clock, with initial value of $36.0 \mathrm{kHz}$, was changed in eight $400-\mathrm{Hz}$ steps. The plots show that total position changes of 2.5 and 16 microns were observed in the horizontal and vertical positions, respectively, with total change of $3.2 \mathrm{kHz}$ in the external clock. This is a significant amount of change in the DC position during the tuning process and needs to be taken into account. Once the tuning is complete, such changes are lumped into BPM offsets, ensuring that the adjusted position readbacks remain unaffected.

\section{AC Effects}

The AC data were measured by a real-time signal analyzer (LDS Dactron Focus II) in the laboratory environment at three external clock values $(35.5 \mathrm{kHz}, 36$ $\mathrm{kHz}$, and $36.5 \mathrm{kHz}$ ) in order to find the impact of the external clock on the Nbbpm module's AC property in a frequency band of $100 \mathrm{~Hz}$. Figure 5 shows the cumulative rms motion at the above-mentioned three clock values. As observed, the external clock variation does not impact the AC data of Nbbpm module, indicating that the external clock can be tuned out without any impact.

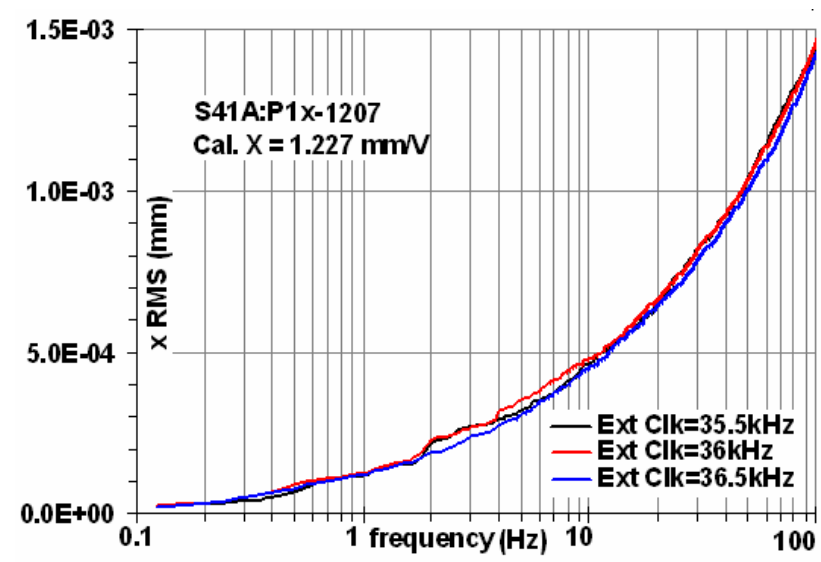

Figure 5: Horizontal AC data is shown for an Nbbpm module measured with a real-time signal analyzer at different external clock frequencies on the PAS9782.

\section{TUNING THE EXTERNAL CLOCK}

Two examples of the tuning procedures and results are discussed here.

\section{Tuning with RF Signal Inputs in the Laboratory}

We measured the AC data of four Nbbpm modules in a laboratory setup with the beam signal simulated by a generator. The cumulative rms data are shown in Figure 6. The AC data plots, with internal clocks, show large steps due to aliasing effects. Then the external clock was applied to all four modules. The external clock was tuned until the steps in all four plots were moved out of the 100$\mathrm{Hz}$ frequency range.

When the external clock was not applied, the Nbbpm worked with internal clocks. Normally, these clocks are of 


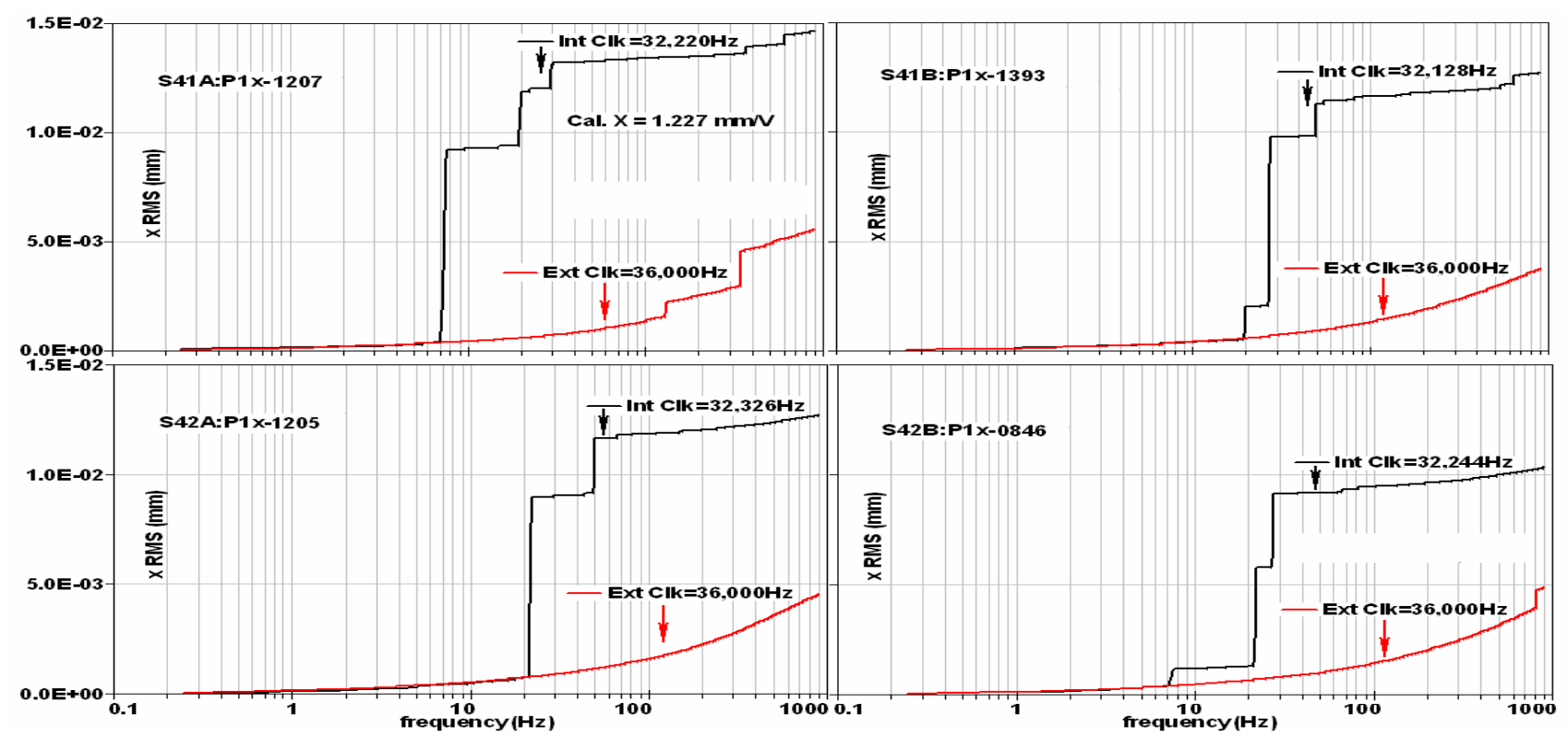

Figure 6: The cumulative rms curves of four Nbbpm modules with and without the external clock at one chassis in the lab. Data is for the horizontal plane and was collected by a real-time signal analyzer.

the order of $32 \mathrm{kHz}$, but with different values. The different internal clocks beat with each other and with other processing clocks, contributing to the steps in the cumulative rms data. When we used one external clock to override all four internal clocks, the cumulative rms data were much smaller. The use of one clock for all four modules was helpful in reducing the rms noise level.

\section{Tuning with Beam Signal}

The external clock, using the PAS9782 module, was employed at Sector 25 in the APS storage ring during the hybrid mode operation with $102-\mathrm{mA}$ beam. The cumulative horizontal rms of Nbbpm S25A:P1 was measured with its internal clock and then with the external clock. The test results are shown in Figure 7 with the internal clock and three different external clocks during the tuning process. The external clock was varied through EPICS control.

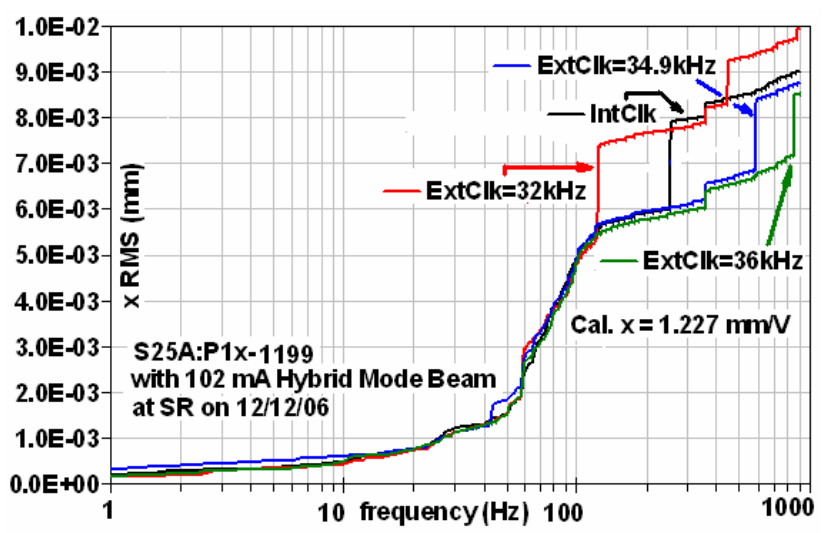

Figure 7: The cumulative rms of BPM S25A:P1 on the $\mathrm{x}$ plane by real-time signal analyzer with an internal and an external clocks that was shifted.

06 Instrumentation, Controls, Feedback \& Operational Aspects
As observed, the step seen in the cumulative rms data at $\sim 250 \mathrm{~Hz}$ (internal clock data) has been moved out to $>$ $800 \mathrm{~Hz}$ by tuning the external clock. After shifting out the step, this BPM now is available for both real-time orbit feedback and as well as for rms calculation.

\section{SUMMARY}

The external clock on the PAS9782 module is much more stable than the internal clocks of the Nbbpm modules. The Nbbpm module AC data is independent from the external clock shift at low band $(0-100 \mathrm{~Hz})$. However, there is a DC data shift with external clock changes. This change is taken into account in the BPM offset value, ensuring no effect on orbit measurement accuracy. The programmable external clock can move the aliasing errors out of the active frequency band of the RTFB system and rms beam motion calculation. One external clock also improves the BPM electronic noise level on the Nbbpm module as compared to using the internal clocks.

\section{ACKNOWLEDGEMENTS}

We thank G. Decker, F. Lenkszus and S. Xu for their support and discussion.

This work is supported by the U. S. Department of Energy, Office of Science, Office of Basic Energy Sciences, under Contract No. DE-AC02-06CH11357.

\section{REFERENCES}

[1] J. Carwardine, G. Decker, K. Evans Jr., A. Hillman, F. Lenkszus, R. Merl and A. Pietryla, "Commissioning of the APS Real-time Orbit Feedback System," PAC'97, Vancouver, B.C., Canada, May 1997, p. 2281, http://www.jacow.org.

[2] PAS 9782/GEN Engineering Specification, Revision B, Precision Analog Systems Co., Plantation, Florida. 